\title{
Localization of infraorbital foramen and accessory infraorbital foramen with reference to facial bony landmarks: predictive method and its accuracy
}

\author{
Jiraporn Suntiruamjairucksa, Vilai Chentanez \\ Department of Anatomy, Faculty of Medicine, Chulalongkorn University, Bangkok, Thailand
}

\begin{abstract}
The infraorbital nerve block is used for mid-facial anesthesia. We aim to determine the location of infraorbital foramen (IOF) and accessory infraorbital foramen (AIOF) with reference to anterior nasal spine (ANS) and the lowest point of zygomaticomaxillary junction $(\mathrm{Z})$ and assess the accuracy of the predictive method. Two hundred and sixteen dry skulls were examined. A reference line was drawn from ANS to Z (line A) and its length was measured (distance A). The location of IOF was predicted by using the mean vertical distance from IOF to line A (line B) which was $15.14 \pm 1.99 \mathrm{~mm}$ and the mean ratio of the distance between ANS and the intersecting point of line B and line A (distance D) to distance A (D:A) which was $63.35 \% \pm 3.90 \%$. Eighty-six AIOFs were found. Most of them located superomedial to IOF, except for 3 AIOFs which located in the inferolateral position. For localization the AIOF, the mean vertical distance was $19.34 \pm 3.36 \mathrm{~mm}$ and the mean ratio was $51.8 \% \pm 5.90 \%$. No statistically significant difference of the predicted distances for both foramens was found between sex and sides. The accuracy of the predictive method was assessed in 15 embalmed cadavers. Predicted IOFs were 50\% accurate and the mean distance error of the predicted IOF was $1.10 \pm 1.44 \mathrm{~mm}$ lateral and $0.59 \pm 1.39 \mathrm{~mm}$ inferior to the exact IOF. Therefore, this study provides an alternative method for localization of IOF and AIOF which could be useful in clinical settings.
\end{abstract}

Key words: Anatomic landmarks, Nerve block, Reference standards, Skull

Received October 20, 2021; Revised November 30, 2021; Accepted December 3, 2021

\section{Introduction}

The infraorbital nerve (ION) derives from the maxillary nerve and emerges from infraorbital foramen (IOF) on the maxilla bone [1]. This nerve provides mid-facial sensation around lower eyelids, lateral side of nose, nasal septum, and upper lips $[2,3]$. The ION block is used for regional anesthe-

\footnotetext{
Corresponding author:

Vilai Chentanez (iD

Department of Anatomy, Faculty of Medicine, Chulalongkorn University, Bangkok 10330, Thailand

E-mail: fmedvct@gmail.com
}

sia in many procedures including maxillofacial, eye, nose, and dental surgeries [4-7]. Moreover, it is beneficial in treating intractable infraorbital neuralgia $[8,9]$ and reducing postoperative pain $[5,10,11]$. Localization of IOF is essential to perform the ION block. In addition to IOF, knowledge of accessory infraorbital foramen (AIOF), a variation of IOF, is also important because neurovascular structures emerging from AIOF, accessory infraorbital nerve (AION), and accessory infraorbital artery (AIOA), were associated with structures from IOF [12]. The infraorbital margin was widely used to predict the location of IOF. The location of IOF was described to be $8-10 \mathrm{~mm}$ below the infraorbital margin in the mid-pupillary line [13]. However, this distance varied between studies and could range from 3.2-13.2 mm [14, 15]. 
There was a failure rate of $17 \%$ when performing the ION block by this method of IOF identification [16]. Previous studies examined various bony and soft tissue landmarks to identify the location of IOF and AIOF, for example, the piriform aperture, zygomaticomaxillary suture, ala nasi, lateral palpebral commissure and lacrimal caruncle [17-23]. These studies observed landmarks which can be examined either on skulls which cannot be palpated on the face of patients or soft tissue landmarks that cannot identified on skulls and some of them were not applicable in practice.

Therefore, in this study, we aimed to determine the location of IOF and AIOF using bony landmarks that can be easily palpated both in skulls and soft tissue which are the anterior nasal spine (ANS) and the lowest point of the zygomaticomaxillary junction (Z). Prevalence of AIOF, anatomical relationship between IOF and AIOF, and accuracy of the proposed predictive method in cadavers were assessed.

\section{Materials and Methods}

A total of 216 human dry skulls and 15 embalmed cadaveric heads were obtained from the Department of Anatomy, Faculty of Medicine, Chulalongkorn University, Thailand. Samples with facial distortions were excluded. Skulls of undetermined age and sex were obtained, the general size and architecture, supraorbital ridges, mastoid process, and occipital protuberance were used for sex determination of skulls. Male skulls are larger in size, have more prominent supraorbital ridges and occipital protuberance with a long and broad mastoid process [24-26]. To avoid bias from one observer, two observers examined all skull separately and a consensus was made if there was any conflict. Therefore, with this process, 153 male and 63 female skulls were determined. This study was approved by the Faculty of Medicine, Chulalongkorn University IRB committee (IRB no.698/64). Data collection was performed cautiously with a structured procedure. This study had two major steps: analysis of skulls and cadaveric dissection.

\section{Analysis of the skulls}

\section{Prevalence, number and position of AIOF}

Skulls were examined by direct visual inspection to find the existence and number of AIOF. The position of AIOF (medial/lateral/superior/inferior) relative with IOF was also observed.

\section{Determination of IOF and AIOF location under stereoscopic} microscope

Skulls were arranged in the Frankfurt horizontal plane and anteroposterior position under the Leica M50 Stereoscopic Microscope (Leica Camera AG, Wetzlar, Germany) with a total magnification of $1.97 \times$. The location of ANS and $\mathrm{Z}$ were identified by palpation under the live image. All measurements were performed under the live image in the Leica Application Suite Core V4.12 (Leica Camera AG). This software provides real-time imaging from the microscope for adjustment and measurement. An imaginary line between ANS and Z was created as line A. Several parameters were examined in each skull including distance from ANS to $\mathrm{Z}$ (distance $\mathrm{A}$ ), vertical distance from middle-upper edge of IOF to line A (distance B), and distance between ANS and intersecting point of the vertical line from IOF with line A (distance D) (Fig. 1). If AIOF existed, vertical distance from middle-upper edge of AIOF to line A (distance C), distance between ANS and intersecting point of the vertical line

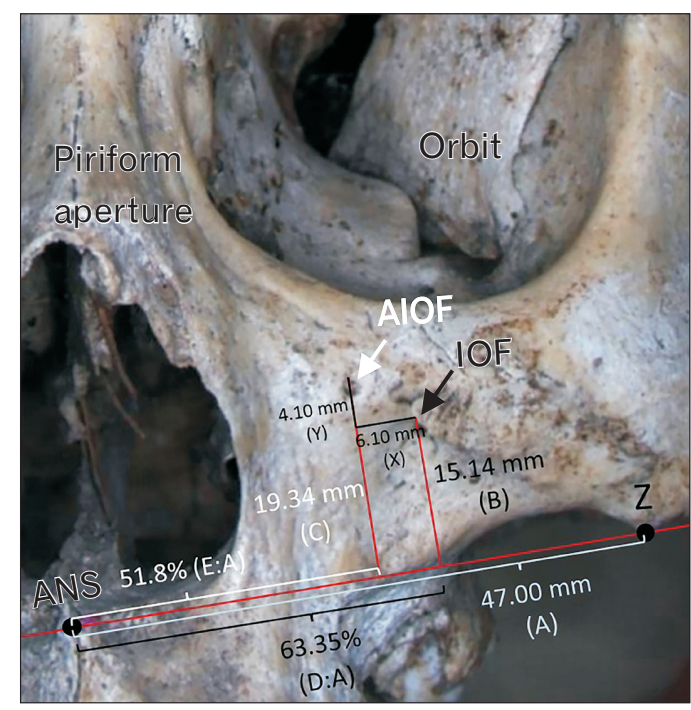

Fig. 1. Picture of skull illustrates the value of main parameters of IOF and AIOF with reference to the horizontal line from ANS to Z (unit: millimeters). A, distance from ANS to Z; AIOF, accessory infraorbital foramen; ANS, anterior nasal spine; $B$, vertical distance from the middle-upper edge of IOF to the line A; $\mathrm{C}$, vertical distance from the middle-upper edge of AIOF to line $\mathrm{A}$; $\mathrm{D}$, horizontal distance from ANS to the intersecting point of the vertical line from IOF with line A; $\mathrm{D}$ : A, percentage of the ratio of distance $\mathrm{D}$ to distance $\mathrm{A}$; E, horizontal distance from ANS to the intersecting point of the vertical line from AIOF with line A; E:A, percentage of the ratio of distance $\mathrm{E}$ to distance A; IOF, infraorbital foramen; X, horizontal distance between IOF and AIOF; Y, vertical distance between IOF and AIOF; Z, the lowest point of zygomaticomaxillary junction. 
from middle-upper edge of AIOF with line A (distance E), the horizontal distance between IOF and AIOF (X) and the vertical distance between IOF and AIOF $(\mathrm{Y})$ were measured (Fig. 1). The predictive method for localization of IOF was generated by using the mean distance $B$ and ratio of the distance D to A (D:A), whereas location prediction of AIOF was determined by the mean distance $\mathrm{C}$ and ratio of the distance E to A (E:A). The prediction of IOF and AIOF was done in embalmed cadavers to assess the accuracy of our proposed method.

\section{Accuracy assessment in cadaveric dissection}

Fifteen cadavers were dissected and the existence and number of AIOF were observed. Position of the predicted

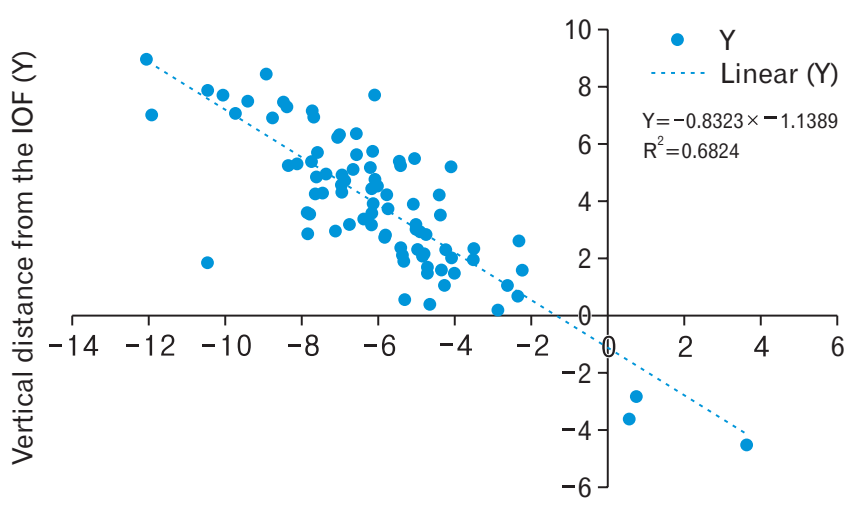

Horizontal distance from the IOF $(X)$

Fig. 2. Scatter plotted graph shows the location of the accessory infraorbital foramen (blue dot) with reference to the infraorbital foramen. AIOF, accessory infraorbital foramen; IOF, infraorbital foramen; X, horizontal distance between IOF and AIOF; Y, vertical distance between IOF and AIOF. foramen in relation with the exact foramen in cadavers (medial/lateral/inferior/superior) were recorded. The shortest horizontal and vertical distance from the predicted IOF to the exact IOF (F and G) and the shortest horizontal and vertical distance from the predicted AIOF to the exact AIOF ( $\mathrm{H}$ and I) were measured directly by a digital Vernier Caliper (Mitutoyo $^{\circledR}$ [Mitutoyo Corp., Kawasaki, Japan] 0-150 mm; range $150 \mathrm{~mm}$, resolution $0.01 \mathrm{~mm}$ ). All measurements were executed twice by a single investigator to minimize intra and inter observer error.

\section{Statistical analysis}

Collected data were analyzed in the IBM SPSS version 22.0 (IBM Corp., Armonk, NY, USA). Frequency and mean with standard deviation were analyzed in each parameter, paired $t$-test analysis was used to assess the mean difference between sides and unpaired $t$-test analysis was used to assess the mean difference between sex. A $P$-value of less than 0.05 was defined as statistically significant. Intra-observer reliability was calculated for each parameter and the least intraobserver intraclass correlation coefficient among all parameters was 0.987 .

\section{Results}

\section{Prevalence, number, and location of AIOF}

The prevalence of AIOF was 19.91\% (86 AIOFs from both sides of 216 skulls) which located on the left side for 46 foramens (53.49\%) and on the right side for 40 foramens (46.51\%). A single AIOF was found in 82 sides. Double AIOFs were found in two skulls presented on the left side and right side.

Table 1. Distance of A, B, C, D, E, X, and Y from image analysis of skulls

\begin{tabular}{|c|c|c|c|c|c|c|c|}
\hline \multirow{2}{*}{ Parameter (mm) } & \multicolumn{3}{|c|}{ Side } & \multicolumn{3}{|c|}{ Sex } & \multirow{2}{*}{ Total } \\
\hline & Left & Right & $P$-value & Male & Female & $P$-value & \\
\hline A & $47.04 \pm 2.96$ & $46.97 \pm 2.94$ & 0.711 & $47.46 \pm 2.90$ & $45.90 \pm 2.78$ & $<0.001^{\star}$ & $47.00 \pm 2.95$ \\
\hline B & $15.12 \pm 2.03$ & $15.16 \pm 1.95$ & 0.711 & $15.18 \pm 1.94$ & $15.05 \pm 2.11$ & 0.533 & $15.14 \pm 1.99$ \\
\hline $\mathrm{C}$ & $19.42 \pm 3.20$ & $19.25 \pm 3.57$ & 0.200 & $19.50 \pm 3.13$ & $18.98 \pm 3.87$ & 0.510 & $19.34 \pm 3.36$ \\
\hline $\mathrm{D}$ & $29.81 \pm 2.35$ & $29.69 \pm 2.29$ & 0.481 & $30.06 \pm 2.25$ & $29.00 \pm 2.32$ & $<0.001^{\star}$ & $29.75 \pm 2.32$ \\
\hline $\mathrm{E}$ & $24.62 \pm 2.66$ & $24.16 \pm 3.59$ & 0.483 & $24.60 \pm 3.26$ & $23.95 \pm 2.73$ & 0.374 & $24.41 \pm 3.11$ \\
\hline $\mathrm{X}$ & $5.91 \pm 2.04$ & $6.32 \pm 2.38$ & 0.837 & $6.22 \pm 2.38$ & $5.83 \pm 1.72$ & 0.453 & $6.10 \pm 2.20$ \\
\hline $\mathrm{Y}$ & $4.00 \pm 2.13$ & $4.22 \pm 2.01$ & 0.214 & $4.08 \pm 2.24$ & $4.16 \pm 1.62$ & 0.845 & $4.10 \pm 2.06$ \\
\hline D:A (\%) & $63.42 \pm 4.02$ & $63.26 \pm 3.79$ & 0.725 & $63.40 \pm 4.00$ & $63.21 \pm 3.66$ & 0.662 & $63.35 \pm 3.90$ \\
\hline E:A (\%) & $52.40 \pm 5.27$ & $51.11 \pm 6.56$ & 0.753 & $51.54 \pm 6.35$ & $52.41 \pm 4.77$ & 0.534 & $51.80 \pm 5.90$ \\
\hline
\end{tabular}

Values are presented as mean \pm SD. A, distance from anterior nasal spine (ANS) to the lowest point of the zygomaticomaxillary junction; $\mathrm{B}$, vertical distance from the middle-upper edge of infraorbital foramen (IOF) to line A; C, vertical distance from the middle-upper edge of accessory infraorbital foramen (AIOF) to line A; D, horizontal distance from ANS to the intersecting point of the vertical line from IOF with line A; E, horizontal distance from ANS to the intersecting point of the vertical line from AIOF with line A; X, horizontal distance between IOF and AIOF; Y, vertical distance between IOF and AIOF; D:A, percentage of the ratio of distance $\mathrm{D}$ to distance $\mathrm{A}$; E:A, percentage of the ratio of distance $\mathrm{E}$ to distance $\mathrm{A}$. ${ }^{\star}$ Statistically significant difference between groups. 
No triple AIOF was observed in this study. There were 21 from 42 skulls (50.0\%) of which AIOF presented bilaterally. Regarding to sex, the prevalence of AIOF was $19.61 \%$ (60 from 306 sides) and 20.63\% (26 from 126 sides) in male and female skulls, respectively. Most of the AIOFs located superomedial to IOF except for 3 AIOFs which located in the inferolateral position (Fig. 2). AIOF located $6.10 \pm 2.20 \mathrm{~mm}$ medial (X) and 4.10 $\pm 2.06 \mathrm{~mm}$ superior (Y) to IOF (Fig. 1).

\section{Predictive method for localization of IOF and AIOF}

The mean distances of A, B, C, D and E were 47.00 \pm 2.95 , $15.14 \pm 1.99,19.34 \pm 3.36,29.75 \pm 2.32$, and $24.41 \pm 3.11 \mathrm{~mm}$, respectively. The mean ratio D:A was $63.35 \% \pm 3.90 \%$ and the ratio E:A was $51.80 \% \pm 5.90 \%$ (Fig. 1). There were statistically significant differences between sex in the distance A and D $(P<0.05)$, other parameters had no statistically significant difference between sex and sides. Detailed information of each parameter is shown in Table 1 . We used the mean ratio of distance $\mathrm{D}$ to $\mathrm{A}(\mathrm{D}: \mathrm{A})$ and the mean distance $\mathrm{B}$ to predict the location of IOF, whereas the mean ratio of distance $\mathrm{E}$ to $\mathrm{A}(\mathrm{E}: \mathrm{A})$ and the mean distance $\mathrm{C}$ were used as the predictive method for localization of AIOF.

\section{Accuracy assessment of the predictive method in cadavers}

Dissection and measurement were performed in $15 \mathrm{ca}-$ davers ( 8 male and 7 female). From 30 IOFs in cadavers, the predicted IOF was found accurately in the same position with the exact IOF for 15 sides (50.0\%) (Fig. 3). There were 10 (33.33\%), $3(10.0 \%)$ and $2(6.67 \%)$ of the predicted IOF that located lateral, inferior, and inferolateral to the exact IOF, respectively. The mean error distance of the predicted IOF was $1.10 \pm 1.44 \mathrm{~mm}(\mathrm{~F})$ lateral and $0.59 \pm 1.39 \mathrm{~mm}$ inferior $(\mathrm{G})$ to the exact IOF.

There were only two single AIOFs that were identified on the left side of a male and a female cadaver. The prevalence of AIOF in the cadaveric study was $6.67 \%$. All predicted AIOFs located lateral to the exact AIOF and the mean horizontal distance error $(\mathrm{H})$ was $1.83 \pm 0.15 \mathrm{~mm}$. No vertical distance

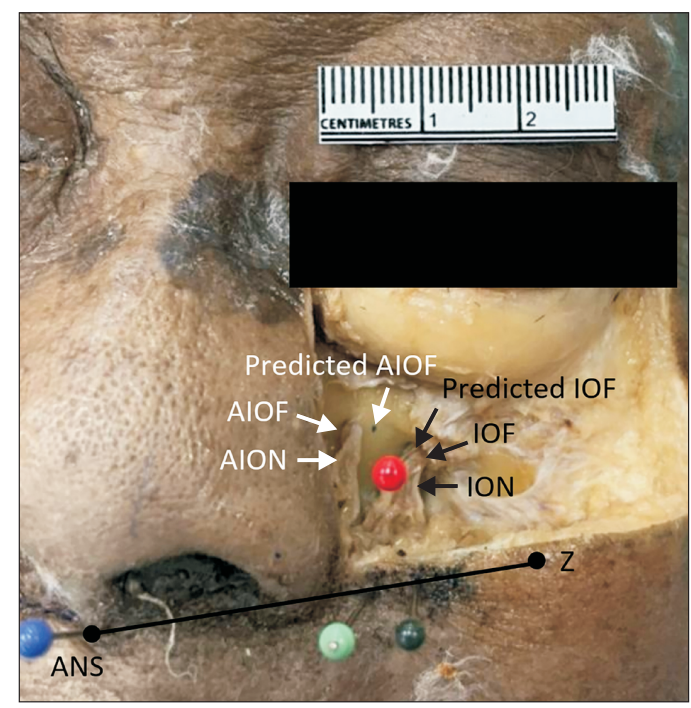

Fig. 3. Picture of left midface of a male cadaver showing the same location of both predicted IOF and the exact IOF (tip of red pin). The predicted AIOF was lateral to the exact AIOF. Dark green pin, location of the predicted distance D (distance A multiplied with the percentage of $\mathrm{D}: \mathrm{A})$; light green pin, location of the predicted distance $\mathrm{E}$ (distance A multiplied with the percentage of $\mathrm{E}: \mathrm{A}$ ); red pin, location of the predicted IOF; blue dot, the location of the predicted AIOF; blue pin, location of the anterior nasal spine. AIOF, accessory infraorbital foramen; AION, accessory infraorbital nerve; ANS, anterior nasal spine; IOF, infraorbital foramen; ION, infraorbital nerve; $\mathrm{Z}$, the lowest point of zygomaticomaxillary junction; black line, the line between ANS and the lowest point of zygomaticomaxillary junction.

Table 2. Distance of A, predicted D, predicted E, F, G, H, and I from cadavers

\begin{tabular}{|c|c|c|c|c|c|c|c|}
\hline \multirow{2}{*}{ Parameter (mm) } & \multicolumn{3}{|c|}{ Side } & \multicolumn{3}{|c|}{ Sex } & \multirow{2}{*}{ Total } \\
\hline & Left & Right & $P$-value & Male & Female & $P$-value & \\
\hline A & $47.29 \pm 2.32$ & $46.86 \pm 2.25$ & 0.117 & $47.22 \pm 2.86$ & $46.91 \pm 1.36$ & 0.705 & $47.07 \pm 2.26$ \\
\hline Predicted D & $29.96 \pm 1.47$ & $29.69 \pm 1.42$ & 0.117 & $29.91 \pm 1.81$ & $29.72 \pm 0.86$ & 0.706 & $29.82 \pm 1.43$ \\
\hline Predicted E & $24.49 \pm 1.20$ & $24.27 \pm 1.17$ & 0.117 & $24.46 \pm 1.48$ & $24.30 \pm 0.70$ & 0.704 & $24.38 \pm 1.17$ \\
\hline $\mathrm{F}$ & $0.64 \pm 1.16$ & $1.56 \pm 1.57$ & $0.019^{*}$ & $1.11 \pm 1.38$ & $1.09 \pm 1.55$ & 0.979 & $1.10 \pm 1.44$ \\
\hline G & $0.40 \pm 1.05$ & $0.79 \pm 1.67$ & 0.138 & $0.41 \pm 1.13$ & $0.80 \pm 1.65$ & 0.453 & $0.59 \pm 1.39$ \\
\hline $\mathrm{H}$ & $1.83 \pm 0.15$ & - & - & - & - & - & $1.83 \pm 0.15$ \\
\hline I & 0 & - & - & - & - & - & 0 \\
\hline
\end{tabular}

Values are presented as mean \pm SD. A, distance from anterior nasal spine (ANS) to the lowest point of the zygomaticomaxillary junction; Predicted D, distance A multiplied with the percentage of ratio D:A (63.35\%); Predicted E, distance A multiplied with the percentage of ratio E:A (51.8\%); F, the shortest horizontal distance from the predicted infraorbital foramen (IOF) to the exact IOF; $G$, the shortest vertical distance from the predicted IOF to the exact IOF; H, the shortest horizontal distance from the predicted accessory infraorbital foramen (AIOF) to the exact AIOF; I, the shortest vertical distance from the predicted AIOF to the exact AIOF. * Statistically significant difference between groups. 
error (I) was detected. There was a statistically significant difference between sides in distance $\mathrm{F}(P<0.05)$, other parameters had no statistically significant difference between sex and sides. The values of each parameter are shown in Table 2.

\section{Discussion}

Localization of IOF is essential to achieve anesthesia of the mid-facial region when performing the ION block. In addition to IOF, understanding the AIOF is also important because the AION emerges from this foramen and it is associated with the ION [12]. Although numerous studies examined various landmarks to identify the location of IOF and AIOF, the results varied between studies. We aimed to determine anatomical landmarks for more precise localization of IOF and AIOF by using easily palpable landmarks both in bone and soft tissue. Therefore, the line between ANS and Z was taken into consideration. Moreover, we introduced a direct identification of the bony landmarks and measurement by imaging software under the stereoscopic microscope. The location of IOF was predicted by using the ratio of distance from ANS to the intersecting point of the vertical line from IOF to line A (distance D) and distance A (ratio D:A) as the predicted horizontal distance, and the predicted vertical distance from the middle-upper edge of IOF to line A (distance B). We found that the mean ratio D:A was $63.35 \% \pm 3.90 \%$ which was about two-thirds of distance A and predicted distance B was $15.14 \pm 1.99 \mathrm{~mm}$. For localizing the AIOF, we used the ratio of the distance from ANS to the intersecting point of the vertical line from AIOF to line $\mathrm{A}$ and the distance A (ratio E:A) which was 51.8\% $\pm 5.9 \%$ (about half of the distance $\mathrm{A}$ ) and the predicted vertical distance from the middle-upper edge of AIOF to line A (distance C) which was $19.34 \pm 3.36 \mathrm{~mm}$. There were no statistically significant differences between sex and sides, therefore, we can use the same value in both sex and sides. We analyzed the accuracy of our predictive method by confirming locations of IOF and AIOF in cadavers. The result showed that the prediction of IOF was $50 \%$ accurate in cadavers. Some of the predicted IOFs were found to be inferior and lateral to the exact IOF. In cadaveric dissection, only two AIOFs were identified. Therefore, there might be too little information to draw a conclusion. However, the mean distance error between predicted and exact foramina ranged from $1-2 \mathrm{~mm}$ which is small and might not be affected by palpation with fingertips in clinical situations. It can be assumed that this predictive method is accurate and clinically applicable for locating IOF and AIOF.

This study provides detailed information about the AIOF. Prevalence of AIOF was $19.91 \%$ in dry skulls and $6.67 \%$ in cadavers which was consistent with the systematic review describing that the prevalence of AIOF in skulls and cadavers ranged from $0.8 \%$ to $27.3 \%$ [27]. On the other hand, later studies found higher prevalence of $32.1 \%$ [28] and 35\% in dry skulls [29]. These cadaveric results were also inconsistent with later studies which found the frequency of AIOF to be $36.7 \%$ [12]. We found that AIOF was commonly a single foramen. There were double AIOFs in 2.4\% of skulls and no triple foramen was found. Previous studies reported that AIOF was mostly a single foramen $[27,29,30]$ and more common on the left side [20, 27-29, 31-33] similar to our findings. There were $50 \%$ of skulls that had bilateral AIOFs in the present study which was higher than previous reports of AIOF occurring bilaterally ranging from $9.1 \%$ to $25 \%$ [27, $28,34,35]$. These differences can be due to differences in population, race, and sample size.

The observations made in this study were consistent with previous reports regarding that most of the AIOFs located superomedial to IOF $[27,32,33]$. However, there were three AIOFs that located in the inferolateral position which was different from previous studies. According to Rai et al. [36] the mean distance from AIOF to IOF was $9.79 \mathrm{~mm}$, whereas, Tezer et al. [33] found that the mean distance between AIOF and IOF was $3.95 \pm 1.6 \mathrm{~mm}$. In this study the mean distance from IOF to AIOF was $6.10 \pm 2.20 \mathrm{~mm}$ in horizontal and $4.10 \pm 2.06 \mathrm{~mm}$ in vertical direction. These discrepancies could be attributed to the method of measurement. The mean distances were measured in two directions separately in our study which differed from previous studies that measured the shortest distance between the IOF and AIOF regarding the direction of the distance.

Some previous studies measured the distance between IOF and ANS, but the method was different from this study. Agthong et al. [20] measured the shortest distance from ANS to the center of IOF (right, 34.1 \pm 0.2 and left, $34.3 \pm 0.2 \mathrm{~mm}$ ) while Chrcanovic et al. [18] measured the shortest distance from the medial-inferior wall of IOF to ANS $(32.38 \pm 2.61$ $\mathrm{mm}$ ). In this study, we measured the distance in two directions, the perpendicular distance from the middle-upper edge of IOF to line A and the horizontal distance from ANS to the intersecting point of the vertical line to line A. This predictive method is more accurate and convenient in practice than measuring the shortest distance from one land- 

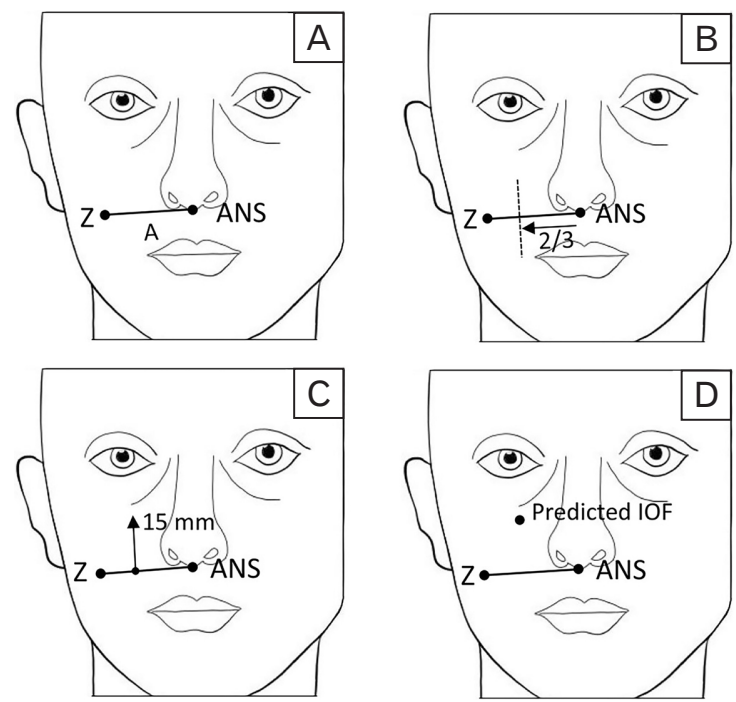

Fig. 4. Illustration describing the predictive method for localization of IOF in clinical practice. (A) Mark the location of ANS at the uppermost part of philtrum at the level of nostrils and $\mathrm{Z}$ at the lowest bony prominence of cheek. Draw line A between ANS and Z. (B) Mark at the medial two-thirds of line A (black dot line). (C) Draw perpendicularly with and above line A at approximately $15 \mathrm{~mm}$. (D) Mark the location of predicted IOF. A, distance from ANS to Z; ANS, anterior nasal spine; IOF, infraorbital foramen; $Z$, the lowest point of the zygomaticomaxillary junction.

mark in which the angle must be considered.

Our approach provides an easy and accurate way for localizing the IOF and AIOF. Clinicians can use this method as an approximation for performing ION block by palpating bony prominences of ANS at the uppermost part of the philtrum at the level of the nostrils of the patient and the lowest bony point of the patient's cheek, then drawing a line between these points (Fig. 4A). Marking at the medial twothirds of that line (Fig. 4B) and drawing perpendicular with and above the line approximately $15 \mathrm{~mm}$ (Fig. 4C), to identify IOF (Fig. 4D). Like IOF, clinicians can mark the point in the middle of the line between the ANS and the lowest bony point of the patient's cheek (Fig. 5B) then measure above that point at approximately $19 \mathrm{~mm}$ (Fig. $5 \mathrm{C}$ ) where AIOF is suspected to be (Fig. 5D).

This study has limitations that should be recognized. Though we identified the sex of each skull by using sex identification criteria, there is no available record of ethnicity, sex and age of skulls which may affect the evaluated results. Moreover, we could not compare our method with the standard method for identifying the IOF in cadavers since an original pupil position in cadavers had changed and could not be identified accurately. For further consideration, in-
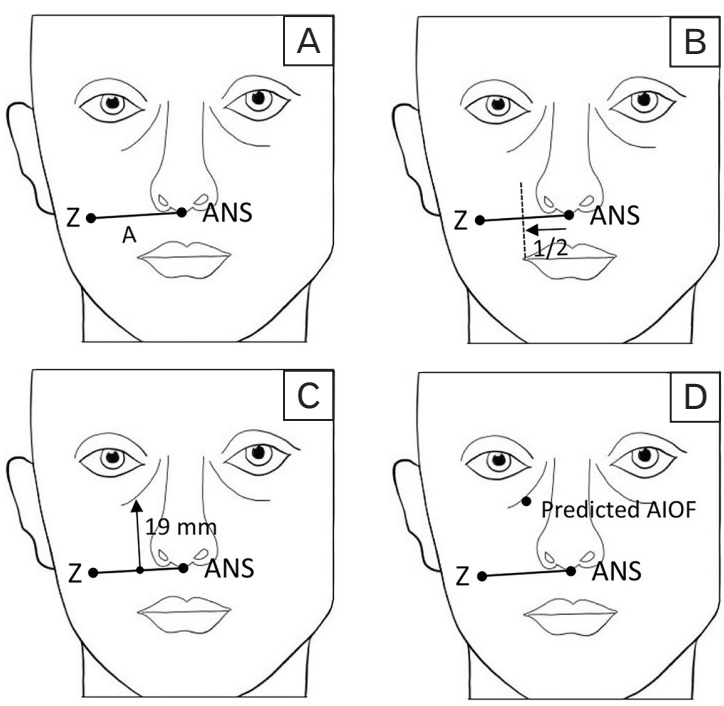

Fig. 5. Illustration describing the predictive method for localization of AIOF in clinical practice. (A) Mark the location of ANS at the uppermost part of philtrum at the level of nostrils and $\mathrm{Z}$ at the lowest bony prominence of cheek. Draw line A between ANS and Z. (B) Mark at the middle of line A (black dot line). (C) Draw perpendicularly with and above line A at approximately $19 \mathrm{~mm}$. (D) Mark the location of predicted AIOF. A, distance from ANS to Z; AIOF, accessory infraorbital foramen; ANS, anterior nasal spine; Z, the lowest point of the zygomaticomaxillary junction.

vestigating the accuracy of this method in patients who underwent the ION block and comparing the results with the standard method should be performed.

In conclusions, we identified the location of IOF and AIOF using ANS and the lowest point of the $\mathrm{Z}$ as reference points. The results of this study provide detailed information of foramina, their relationship and generate the predictive method for localization of IOF and AIOF which is $50 \%$ accurate with a mean distance error of 1-2 $\mathrm{mm}$. The proposed method could be an alternative approach and particularly useful in clinical settings.

\section{ORCID}

Jiraporn Suntiruamjairucksa:

https://orcid.org/0000-0003-4244-506X

Vilai Chentanez: https://orcid.org/0000-0002-5017-5090

\section{Author Contributions}

Conceptualization: JS, VC. Data acquisition: JS. Data analysis or interpretation: JS, VC. Drafting of the manuscript: JS. Critical revision of the manuscript: VC. Approval 
of the final version of the manuscript: all authors.

\section{Conflicts of Interest}

No potential conflict of interest relevant to this article was reported.

\section{Acknowledgements}

The authors would like to express our sincere appreciation to all those who have donated their bodies for medical study and research. Special thanks are extended to the technical staffs of the Department of Anatomy, Faculty of Medicine, Chulalongkorn University for their support in cadaveric care.

\section{References}

1. Elhadi AM, Zaidi HA, Yagmurlu K, Ahmed S, Rhoton AL Jr, Nakaji P, Preul MC, Little AS. Infraorbital nerve: a surgically relevant landmark for the pterygopalatine fossa, cavernous sinus, and anterolateral skull base in endoscopic transmaxillary approaches. J Neurosurg 2016;125:1460-8.

2. Hu KS, Kwak HH, Song WC, Kang HJ, Kim HC, Fontaine C, Kim HJ. Branching patterns of the infraorbital nerve and topography within the infraorbital space. J Craniofac Surg 2006;17:1111-5.

3. Hu KS, Kwak J, Koh KS, Abe S, Fontaine C, Kim HJ. Topographic distribution area of the infraorbital nerve. Surg Radiol Anat 2007;29:383-8.

4. Bösenberg AT, Kimble FW. Infraorbital nerve block in neonates for cleft lip repair: anatomical study and clinical application. $\mathrm{Br}$ J Anaesth 1995;74:506-8.

5. Choi H, Jung SH, Hong JM, Joo YH, Kim Y, Hong SH. Effects of bilateral infraorbital and infratrochlear nerve block on emergence agitation after septorhinoplasty: a randomized controlled trial. J Clin Med 2019;8:769.

6. Molliex S, Navez M, Baylot D, Prades JM, Elkhoury Z, Auboyer C. Regional anaesthesia for outpatient nasal surgery. Br J Anaesth 1996;76:151-3.

7. Spinelli G, Rocchetta D, Carnevali G, Valente D, Conti M, Agostini T. Infraorbital nerve block for isolated orbital floor fractures repair: review of 135 consecutive cases. Plast Reconstr Surg Glob Open 2014;2:e97.

8. Cok OY, Deniz S, Eker HE, Oguzkurt L, Aribogan A. Management of isolated infraorbital neuralgia by ultrasound-guided infraorbital nerve block with combination of steroid and local anesthetic. J Clin Anesth 2017;37:146-8.

9. Perloff MD, Chung JS. Urgent care peripheral nerve blocks for refractory trigeminal neuralgia. Am J Emerg Med 2018;36:205860 .
10. Boselli E, Bouvet L, Augris-Mathieu C, Bégou G, Diot-Junique N, Rahali N, Vertu-Ciolino D, Gérard C, Pivot C, Disant F, Allaouchiche $\mathrm{B}$. Infraorbital and infratrochlear nerve blocks combined with general anaesthesia for outpatient rhinoseptoplasty: a prospective randomised, double-blind, placebo-controlled study. Anaesth Crit Care Pain Med 2016;35:31-6.

11. McAdam D, Muro K, Suresh S. The use of infraorbital nerve block for postoperative pain control after transsphenoidal hypophysectomy. Reg Anesth Pain Med 2005;30:572-3.

12. Iwanaga J, Kikuta S, Kusukawa J, Tomaszewski KA, Walocha JA, Tubbs RS. Anatomic study of accessory infraorbital nerves and foramina: application for a better understanding of complications of le fort fractures and osteotomy. J Oral Maxillofac Surg 2020;78:717-23.

13. Blanton PL, Jeske AH. The key to profound local anesthesia: neuroanatomy. J Am Dent Assoc 2003;134:753-60.

14. Gupta T. Localization of important facial foramina encountered in maxillo-facial surgery. Clin Anat 2008;21:633-40.

15. Singh R. Morphometric analysis of infraorbital foramen in Indian dry skulls. Anat Cell Biol 2011;44:79-83.

16. Pascal J, Charier D, Perret D, Navez M, Auboyer C, Molliex S. Peripheral blocks of trigeminal nerve for facial soft-tissue surgery: learning from failures. Eur J Anaesthesiol 2005;22:480-2.

17. Kazkayasi M, Ergin A, Ersoy M, Bengi O, Tekdemir I, Elhan A. Certain anatomical relations and the precise morphometry of the infraorbital foramen--canal and groove: an anatomical and cephalometric study. Laryngoscope 2001;111(4 Pt 1):609-14.

18. Chrcanovic BR, Abreu MH, Custódio AL. A morphometric analysis of supraorbital and infraorbital foramina relative to surgical landmarks. Surg Radiol Anat 2011;33:329-35.

19. Aggarwal A, Kaur H, Gupta T, Tubbs RS, Sahni D, Batra YK, Sondekoppam RV. Anatomical study of the infraorbital foramen: a basis for successful infraorbital nerve block. Clin Anat 2015;28:753-60.

20. Agthong S, Huanmanop T, Chentanez V. Anatomical variations of the supraorbital, infraorbital, and mental foramina related to gender and side. J Oral Maxillofac Surg 2005;63:8004.

21. Song WC, Kim SH, Paik DJ, Han SH, Hu KS, Kim HJ, Koh KS. Location of the infraorbital and mental foramen with reference to the soft-tissue landmarks. Plast Reconstr Surg 2007;120:1343-7.

22. Zheng WX, Guo JL, Song BX, Liu XL, Lv DL, Tian Y, Li YQ, Cheng FB. Location of the supraorbital and infraorbital foramen with references to the soft tissue landmarks in a Chinese population. J Craniofac Surg 2012;23:1154-5.

23. Ercikti N, Apaydin N, Kirici Y. Location of the infraorbital foramen with reference to soft tissue landmarks. Surg Radiol Anat 2017;39:11-5.

24. Pickering RB, Bachman D. The use of forensic anthropology. Boca Raton: CRC Press; 1997. p. 84-6.

25. Williams BA, Rogers T. Evaluating the accuracy and precision of cranial morphological traits for sex determination. J Forensic Sci 2006;51:729-35. 
26. White TD, Folkens PA. The human bone manual. Amsterdam: Elsevier Academic; 2005. p. 388-9.

27. Hwang K, Lee SJ, Kim SY, Hwang SW. Frequency of existence, numbers, and location of the accessory infraorbital foramen. J Craniofac Surg 2015;26:274-6.

28. Polo CL, Abdelkarim AZ, von Arx T, Lozanoff S. The morphology of the infraorbital nerve and foramen in the presence of an accessory infraorbital foramen. J Craniofac Surg 2019;30:24453.

29. Nam Y, Bahk S, Eo S. Anatomical study of the infraorbital nerve and surrounding structures for the surgery of orbital floor fractures. J Craniofac Surg 2017;28:1099-104.

30. Dagistan S, Miloglu Ö, Altun O, Umar EK. Retrospective morphometric analysis of the infraorbital foramen with cone beam computed tomography. Niger J Clin Pract 2017;20:1053-64.

31. Gour KK, Nair S, Trivedi GN, Gupta SD. Anthropometric measurements of infra orbital foramen in dried human skulls. Int J Biol Med Res 2012;3:2003-6.
32. Martins-Júnior PA, Rodrigues CP, De Maria ML, Nogueira LM, Silva JH, Silva MR. Analysis of anatomical characteristics and morphometric aspects of infraorbital and accessory infraorbital foramina. J Craniofac Surg 2017;28:528-33.

33. Tezer M, Öztürk A, Akgül M, Gayretli Ö, Kale A. Anatomic and morphometric features of the accessory infraorbital foramen. J Morphol Sci 2011;28:95-7.

34. Ali IK, Sansare K, Karjodkar FR, Salve P. Cone beam computed tomography assessment of accessory infraorbital foramen and determination of infraorbital foramen position. J Craniofac Surg 2018;29:e124-6.

35. Shin KJ, Lee SH, Park MG, Shin HJ, Lee AG. Location of the accessory infraorbital foramen with reference to external landmarks and its clinical implications. Sci Rep 2020;10:8566.

36. Rai AR, Rai R, Vadgaonkar R, Madhyastha S, Rai RK, Alva D. Anatomical and morphometric analysis of accessory infraorbital foramen. J Craniofac Surg 2013;24:2124-6. 